\title{
Protection against poliomyelitis in Europe
}

H Nokleby (hanne.nokleby@ecdc.europa.eu) ${ }^{1}$, H De Carvalho Gomes ${ }^{1}$, K Johansen ${ }^{1}$, P Kreidl ${ }^{1}$

1. European Centre for Disease Prevention and Control (ECDC), Stockholm, Sweden

Citation style for this article:

Citation style for this article: Nokleby H, De Carvalho Gomes H, Johansen K, Kreidl P. Protection against poliomyelitis in Europe. Euro Surveill.

2010;15(17): pii=19556. Available online: http://www.eurosurveillance.org/ViewArticle.aspx?Articleld=19556

This article has been published on 29 April 2010

The reappearance of circulating wild poliovirus type 1 (WPV 1) in Tajikistan is the first outbreak from imported wild poliovirus since the World Health Organization (WHO) European Region was declared polio-free in 2002. The risk of poliomyelitis importation to the European Union and European Economic Area countries has probably not increased, but the current outbreak is a reminder that high vaccination coverage, monitoring of protective immunity and maintaining surveillance are important to sustain the present polio-free situation.

Poliomyelitis (polio) is an acute, communicable disease caused by one of three wild-type poliovirus serotypes (WPV types 1-3), or by vaccine associated paralytic polio (VAPP) caused by the live, oral vaccine (OPV). It is characterised by symptoms of varying degree of severity, from subclinical or non-specific disease to rapid onset of acute flaccid paralysis (AFP). The polioviruses are spread mostly by the faecal - oral route. Before vaccination was introduced, most children were exposed to wild-type poliovirus. Of the persons infected, 1:100 to $1: 1,000$ develop paralytic polio, depending on age, and with the lowest incidence in the very young. It has been discussed that a genetic factor in the host could play a role in why only some individuals develop paralytic poliomyelitis [1].

The first polio vaccine, an inactivated polio vaccine (IPV), became available in 1955. The number of polio cases decreased rapidly in countries introducing the vaccine. Trivalent live attenuated oral polio vaccine (OPV) was launched in 1963. The OPV elicits mucosal immunity, which makes it more efficient in stopping the spread of virus than IPV. The OPV is also easier to apply as no injections are needed, and the need for educated healthcare personnel is limited. The Global Polio Eradication Initiative (GPEI) was launched by World Health Organization (WHO) in 1988, with the goal of eradicating polio before the year 2000 [2]. The GPEI was based on the availability and use of OPV, making large immunisation campaigns in countries with limited financial and healthcare resources possible, even though the need to maintain an adequate cold chain to avoid potency loss has been a challenge in many countries.
Use of OPV contains a small risk of polio-like disease caused by one of the three Sabin vaccine-related poliovirus serotypes; vaccine associated paralytic polio (VAPP). VAPP is seen after about one of one million vaccinations, most often in immunocompromised individuals. Through replication and spread in a susceptible population, the vaccine virus may gradually change into a vaccine-derived poliovirus (VDPV) and regain virulence (Table 1). Outbreaks caused by circulating vaccine-derived virus have been reported from several countries worldwide, with eg 153 paralytic cases reported from Nigeria (VDPV2) in 2009 [3]. To avoid this risk most European countries now use only IPV in their vaccination programs [4].

\section{The Global Polio Eradication Initiative}

The GPEI has had an enormous impact on the number of polio cases in the world. The total number of cases decreased from an estimated 350,000 in 1988 to less than 2,000 cases in 2009, and the number of polio endemic countries from 125 to four. The criteria for declaring a single country or a whole WHO region poliofree include reporting of zero indigenous polio cases for at least three years, and a documented surveillance system good enough to discover potential cases. The WHO Region of the Americas was declared polio-free in 1994, the Western Pacific Region in 2000 and the European Region in 2002 [5].

In spite of the large decrease in the number of polio cases the goal of global eradication has been difficult to reach. Wild poliovirus type 2 has not been detected since 1999, but types 1 and 3 are both circulating, and are still endemic in Pakistan, Afghanistan, Nigeria, and India. In the first three countries the main problem has been lack of immunisation, due to local vaccination opposition as in the case of Nigeria [6] or immunisation problems in areas of conflict. In India outbreaks with poliovirus 1 and 3 have continued in spite of very high vaccination coverage. The most probable explanation in India is that the OPV has not been sufficiently immunogenic in some population groups. Monovalent type 1 and 3 polio vaccines provide better immunogenicity [7], and in 2009 an almost as immunogenic bivalent vaccine against types 1 and 3 was introduced (Table 2). The number of cases and affected areas in India has recently been reduced due to these efforts. 
Imported infections from the four still endemic countries have been observed in many parts of the world, and in Africa virus circulation has been re-established in some countries that have been polio-free for many years, mostly due to importation from Nigeria since polio again became endemic there in 2003 [8]. However, the general situation has improved recently, with only 71 notified cases in 2010 compared with 328 cases at the same time in 2009 [9].

\section{The current outbreak in Tajikistan - an important reminder for the European Union (EU)/European \\ Economic Area (EEA)}

The present outbreak of polio from a polio serotype 1 virus in Tajikistan is described in another article in this issue of Eurosurveillance [10]. For the EU/ EEA countries this outbreak does probably not change the current risk of polio importation, as there is already much travel between the four large polio-endemic countries and the EU/EEA. However, the situation in Tajikistan is a reminder that importation of poliovirus to polio-free regions may happen at any time as long as polio virus is circulating in the world. In many European countries there may be population pockets with lower vaccination coverage, where introduction of poliovirus can lead to reestablishment of virus circulation. Earlier outbreaks, such as in the Netherlands in 1992, have shown that this may happen, even in countries with high general vaccination coverage [11].

Polio surveillance has several elements, AFP surveillance being one of them. Enhanced enterovirus surveillance is accepted by WHO as an alternative in countries that have been polio-free for years. Moreover, measuring of vaccination coverage and monitoring of protective immunity in the population are tools for controlling whether immunisation efforts lead to the expected result. Another element is checking for poliovirus in the environment, usually done in the form of sewage sampling. Checking defined sewage systems for wild or vaccine-derived poliovirus is performed routinely in seven EU countries and helps to quantify the current risk of virus importation in each country.

The change from OPV to IPV reduces the risk of disease caused by VPDV. WHO still considers use of OPV necessary to control polio in countries where the disease is still endemic or circulation is re-established. Regardless of that, several Indian paediatricians have advocated IPV also in India and trials are currently underway in less privileged populations [12]. Encouraging countries to change to IPV when possible will reduce the risk for VAPP for everybody.

High vaccination coverage in all parts of the population is the most important part of polio protection in Europe. Good surveillance, including enhanced enterovirus or AFP surveillance, vaccination coverage and population immunity, will help us discover weaknesses in the system or eventual importation of poliovirus, and make us able to implement the necessary measures to avoid reestablishment of polio circulation in a timely manner.

As long as poliovirus is circulating anywhere in the world it may easily be imported to polio-free regions. High vaccination coverage, including booster doses of IPV for persons travelling to polio endemic countries, and enhanced surveillance to detect imported cases early is necessary to avoid re-established circulation in other countries.

TABLE 1

Polioviruses that cause paralytic disease

\begin{tabular}{|l|l|}
\hline WPV & Wild poliovirus serotype 1,2 and 3 \\
\hline VPV & Virus identical to the oral polio vaccine (OPV) virus serotype 1,2 and 3 \\
\hline VDPV & Vaccine-derived poliovirus serotype 1,2 and 3 , vaccine-like virus, but gene sequence differing more than $1 \%$ from the vaccine virus \\
\hline CVDPV & Circulating VDPV \\
\hline
\end{tabular}

\section{TABLE 2}

Vaccines against poliomyelitis

\begin{tabular}{|l|l|}
\hline IPV & Trivalent inactivated polio vaccine, used in most industrialised countries \\
\hline OPV & Trivalent live oral attenuated polio vaccine, used for polio control and eradication in most of the world \\
\hline mOPV1 and mOPV 3 & Monovalent oral vaccines against poliovirus serotype 1 or 3 , used in India and some other endemic areas \\
\hline bOPV & Bivalent oral vaccine against poliovirus serotype 1 and 3, recently introduced in India, Pakistan and Nigeria \\
\hline
\end{tabular}




\section{References}

1. Kindberg E, Ax C, Fiore L, Svensson L. Ala67Thr mutation in the poliovirus receptor CD155 is a potential risk factor for vaccine and wild-type paralytic poliomyelitis. J Med Virol. 2009;81(5):933-6.

2. WHA Resolution 41.28. Global eradication of polio by the year 2000 . Available from: http://www.polioeradication.org/ content/WHA.Resolutions.and.Decisions.pdf

3. WHO Global Polio Eradication Initiative. Circulating vaccine derived polioviruses virus 2000-2010. Available from: http:// www.polioeradication.org/content/general/cvdpv_count.pdf

4. EUVAC.NET: Childhood vaccination schedule. Available from: http://www.ssi.dk/graphics/euvac/vaccination/turkey.html

5. Hoile E. WHO declares European Region polio-free. Euro Surveill. 2002;6(27). pii=1932. Available from: http://www. eurosurveillance.org/ViewArticle.aspx?Articleld $=1932$

6. WHO Weekly Epidemiological Record. 2005;80:305-11.

7. Grassly NG, Jafari H, Bahl S, et al. Mucosal Immunity after Vaccination with Monovalent and trivalent Oral Poliovirus Vaccine in India. JID. 2009;200:794-800.

8. WHO Global Alert and Response (GAR). Poliomyelitis. Available from: http://www.who.int/csr/don/archive/disease/ poliomyelitis/en/index.html

9. WHO Global Polio Eradication Initiative. Wild poliovirus Weekly Update. 27 april 2010. Available from: http://www. polioeradication.org/casecount.asp

10. World Health Organization Country Office Tajikistan, World Health Organization Regional Office for Europe, European Centre for Disease Prevention and Control. Outbreak of poliomyelitis in Tajikistan in 2010: risk for importation and impact on polio surveillance in Europe? Euro Surveill. 2010;15(17). pii=19558. Available from: http://www. eurosurveillance.org/ViewArticle.aspx?Articleld =19558

11. Oostvogel PM, van Wijngaarden JK, van der Avoort HG, et al. Poliomyelitis outbreak in an unvaccinated community in The Netherlands, 1992-93. Lancet. 1994;344(8923):630-1

12. John J. Role of injectable and oral polio vaccines in polio eradication. Expert Rev Vaccines. 2009;8(1):5-8. 